\title{
Synergistic Effects of Aldehyde Dehydrogenase 2 Polymorphisms and Alcohol Consumption on Cognitive Impairment after Ischemic Stroke in Han Chinese
}

\author{
Ying Yu $\mathbb{D}^{1,2}$ Jie Gao, ${ }^{1,3}$ Shasha Wang, ${ }^{1,3}$ Heng Lv, ${ }^{4}$ Liping Xiao, ${ }^{4}$ Hengyuan Shi, ${ }^{4}$ \\ and Xianjie Jia ${ }^{4}$ \\ ${ }^{1}$ Department of Physiology, School of Basic Medicine, Bengbu Medical College, Bengbu 233000, China \\ ${ }^{2}$ Department of Physiology and Pathophysiology, School of Basic Medicine, Shandong University, Jinan 250012, China \\ ${ }^{3}$ Key Laboratory of Cardiovascular and Cerebrovascular Diseases, Bengbu Medical College, Bengbu 233030, China \\ ${ }^{4}$ Department of Epidemiology and Statistics, School of Public Health, Bengbu Medical College, Bengbu 233000, China
}

Correspondence should be addressed to Xianjie Jia; xjjia@139.com

Received 1 April 2021; Revised 16 May 2021; Accepted 7 June 2021; Published 25 June 2021

Academic Editor: Lambros Messinis

Copyright ( 92021 Ying Yu et al. This is an open access article distributed under the Creative Commons Attribution License, which permits unrestricted use, distribution, and reproduction in any medium, provided the original work is properly cited.

\begin{abstract}
Aldehyde dehydrogenase $2(A L D H 2)$ polymorphisms are related to both stroke risk and alcohol consumption. However, the influence of $A L D H 2$ polymorphisms and alcohol consumption on cognitive impairment after ischemic stroke remains unknown, as do the possible mechanisms. We enrolled 180 Han Chinese ischemic stroke patients from four community health centers in Bengbu, China. Cognitive function was assessed using the Montreal Cognitive Assessment (MoCA), and two different MoCA cutoff scores were used to define cognitive impairment in ischemic stroke patients. The ALDH2 genotypes were determined using polymerase chain reaction and direct sequencing. To assess the associations of ALDH2 polymorphisms and alcohol consumption with cognitive impairment after ischemic stroke, we performed binary logistic regression analysis with odds ratios. We revealed that individuals with the $A L D H 2$ wild-type genotype were more likely to have high MoCA scores than those with the mutant and heterozygous types $(p=0.034)$. In addition, using two MoCA cutoff scores, the percentage of moderate to excessive alcohol consumption in the cognitive impairment group was higher than that in the nonimpairment group $(p=0.001)$. The levels of 4 hydroxy-2-nonenal $(p=0.001)$ and swallowing function $(p=0.001)$ were also higher in the cognitive impairment group than in the nonimpairment group. Moreover, after adjusting for other potential risk factors, ALDH2 polymorphisms and alcohol consumption had a significant synergistic effect on cognitive impairment $(p=0.022)$. Specifically, the ALDH2 $* 2$ mutant allele and higher alcohol consumption were associated with cognitive impairment and swallowing ability after ischemic stroke. Targeting ALDH2 may be a useful biomarker for cognitive rehabilitation following ischemic stroke.
\end{abstract}

\section{Introduction}

Stroke is an important problem in public health and is the leading cause of adult disability worldwide $[1,2]$. It has been reported that approximately 15 million people per year have a stroke [3]. Most patients experience some disturbance in cognitive function following stroke [4], and cognitive function is a significant focus in stroke rehabilitation [5]. Alcohol consumption, which is common worldwide, affects the development of stroke and cognitive performance [6,7]. Epidemiological evidence has revealed that excessive drinking is a major risk factor for all stroke subtypes, but especially for ischemic stroke [8, 9]. Additionally, some cohort studies have suggested that light to moderate drinking may have a protective effect on cardiovascular disease and ischemic stroke [9, 10]. In contrast, more recent studies have indicated that alcohol consumption is roughly linearly associated with stroke risk $[11,12]$. However, a U-shaped relationship has also been reported between regular alcohol consumption and cognitive function in several major epidemiological studies [13]; thus, the relationship between alcohol consumption and cognitive impairment after ischemic stroke remains uncertain. 
Alcohol metabolism represents a key biological determinant that can impact drinking behavior. Aldehyde dehydrogenase $2(\mathrm{ALDH} 2)$ is the primary enzyme involved in this metabolic process [14]. Previous research has examined the potential effects of ALDH2 on alcohol consumption and health outcomes. However, the relationship between ALDH2, alcohol consumption, and cognitive function in patients with ischemic stroke is unclear. The main function of ALDH2 is to detoxify acetaldehyde, which is a toxic chemical product of ethanol metabolism [15]. Moreover, ALDH2 also removes other toxic aldehydes, such as 4hydroxy-2-nonenal (4-HNE). As a potential substrate of ALDH2, 4-HNE is commonly considered a specific marker of ischemic stroke injury $[15,16]$.

ALDH2 is abundant in the brain, heart, lungs, and other organs with high mitochondrial contents [17, 18]. The $A L D H 2$ gene consists of 13 exons and 12 introns. In exon 12 , a polymorphism exists, in the form of a G-to-A missense mutation. The glutamate at position 504 is substituted by lysine (Glu504Lys). This polymorphism is also known as rs671, or the ALDH2 * 2 form, while the more common wild-type form is known as $A L D H 2 * 1$. There are thus three possible allele combinations in the population: wild-type $(* 1 / * 1)$, heterozygote $(* 1 / * 2)$, and mutant $(* 2 / * 2)$ [19, 20]. Approximately $40 \%$ of the East Asian population carries an $A L D H 2 * 2$ mutant allele, with a resulting marked reduction in enzymatic activity [15]. Recent studies have reported that the Glu504Lys polymorphism may affect ischemic stroke risk in the Han Chinese population and that carriers of the ALDH2 * 2 allele have increased 4-HNE levels after stroke [21]. However, there has been little previous research into the association between $A L D H 2$ genotypes and cognitive impairment after ischemic stroke.

Therefore, the aim of this study was to evaluate the association of $A L D H 2$ genotypes and alcohol consumption with cognitive function after ischemic stroke. Cognitive function can be tested briefly using the Montreal Cognitive Assessment (MoCA), and this test is recommended for screening cognitive impairment in patients with ischemic stroke [22]. In many previous studies, cognitive impairment has been defined as a MoCA score $<26$ [23]. However, some researchers have recommended that a MoCA cutoff score of 22/23 points might be more suitable for detecting cognitive impairment [24]. In addition, swallowing deficits are also commonly reported in patients with ischemic stroke [25], and cognitive dysfunction is related to dysphagia [26]. In the present study, we used these two different MoCA cutoff scores to investigate the relationship between alcohol consumption, 4-HNE levels, swallowing function, and cognitive impairment after ischemic stroke, respectively. We further investigated the synergistic effects of $A L D H 2$ genotype and alcohol consumption on the MoCA score and swallowing ability in ischemic stroke patients. Finally, we sought to explore the underlying mechanisms that might influence these associations.

\section{Methods}

2.1. Patients. From June 2015 through August 2015, patients with ischemic stroke from four community health centers located in the Longzihu District of Bengbu (Anhui Province, China) were recruited in our study. We visited each community and held a free health checkup for participants. Each participant completed a self-reported questionnaire relating to their lifestyle and medical history, including information on prior stroke and baseline disease status. The inclusion criteria for ischemic stroke patients were as follows: (i) stroke diagnosis as per the revised diagnostic criteria of the 4th National Cerebrovascular Disease Conference in China [27], (ii) stroke diagnosis based on computed tomography or magnetic resonance imaging brain scans, (iii) within 3 months after stroke onset, (iv) permanent residents of Han Chinese ethnicity in selected communities, and (v) informed consent provided. The exclusion criteria were as follows: (i) previous history of cerebral vascular malformation, transient ischemic attack, intracranial hemorrhage, stroke mimics (i.e., seizures or migraines), or neurological deficits; (ii) previous history of bleeding diathesis, anticoagulation therapy, illicit drug use, or serious medical illness; (iii) previous history of illiteracy or any major mental or physical condition that may interfere with cognitive assessments; and (iv) a diagnosis of coronary artery disease [19, 22, 28].

Data were initially obtained from 200 participants. We excluded patients who were unable to participate in the interview because of serious cognitive impairment $(n=5)$ and those with missing medical records $(n=4)$. We also excluded subjects who had missing information regarding alcohol habits, such as alcohol status and the amount and frequency of alcohol consumption $(n=7)$, and regarding recurrence and death $(n=4)$. Thus, a total of 180 patients with ischemic stroke were enrolled in this study. A detailed flowchart showing participant selection is provided in Figure 1.

2.2. Demographic and Clinical Characteristics and Measurements. We obtained patient demographic information from patients' medical charts and self-reported data. According to the medical charts, patients were divided into two subtypes using a simple clinical scheme with the Oxfordshire Community Stroke Project (OCSP) classification and included the following: posterior circulation infarction (PCI) and anterior circulation infarction (ACI) [29]. In the PCI group, the infarcts involved the brainstem, posterior cerebral artery area, thalamus, or cerebellum; in the ACI group, the infarcts occurred in the region of the middle cerebral artery, anterior cerebral artery, or anterior choroidal artery [30].

After fasting for 8 to 12 hours, we measured each patient's blood pressure, height, and weight and calculated their body mass index (BMI). In addition, fasting venous blood samples were collected at approximately the same time of day, in the morning, to minimize diurnal variations [31]. Each blood sample was drawn into a tube containing ethylenediaminetetraacetic acid as an anticoagulant and a tube without anticoagulant. The obtained plasma and serum were preserved at $-80^{\circ} \mathrm{C}$ until assays were performed. Routine blood and biochemistry tests were analyzed, including fasting plasma glucose (FPG), total cholesterol (TC), triglycerides (TG), high-density lipoprotein cholesterol (HDL-C), and low-density lipoprotein cholesterol (LDL-C). This part of 


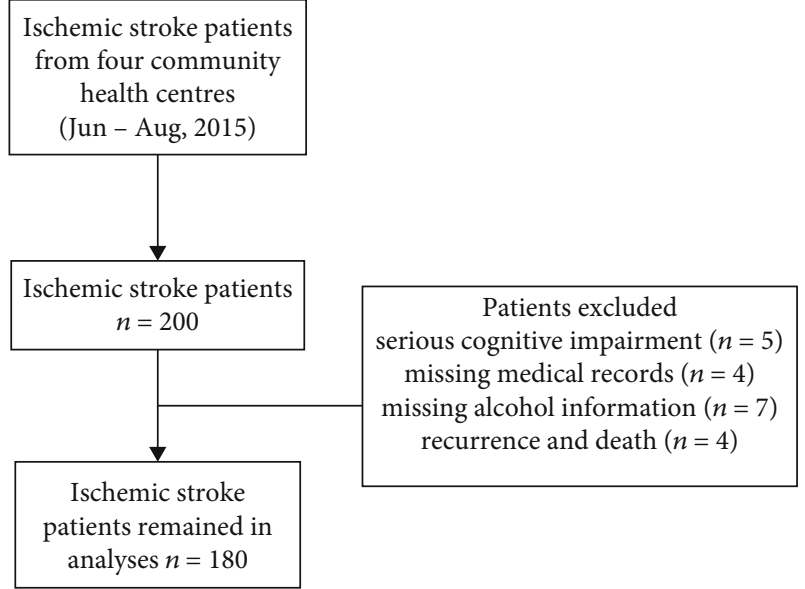

Figure 1: Flowchart of the participant selection process.

the study was approved by the ethics committee of Bengbu Medical College. The cognitive assessments were conducted at the same time as the blood samples were taken.

2.3. Cognitive Assessments. The MoCA is a cognitive screening tool that can be used to distinguish healthy cognitive aging from mild cognitive impairment [32]. It is simple to conduct, sensitive, and valid. Since its introduction into clinical practice, it has been repeatedly demonstrated to be suitable for the initial assessment of mental status and for follow-up assessments [33]. The MoCA was administered by trained physicians in each community.

The MoCA comprises eight subtests that involve visuospatial/executive, naming, memory, attention, language, abstraction, delayed recall, and orientation with respect to time and place. MoCA scores range from 0 to 30. A higher score indicates better cognitive performance [34, 35]. With a cutoff of 26 (which we used as Method 1 in our study), the sensitivity and specificity of MoCA have been reported as $90 \%$ and $87 \%$, respectively, when administered to screen patients with mild cognitive impairment in Canada [32]. However, subsequent clinical studies have demonstrated that some patients with normal cognitive ability have MoCA scores below 26 [36]. The MoCA cutoff scores of ischemic stroke patients by the educational level have been reported as follows: $24 / 25$ for individuals with $\geq 7$ years of education, $19 / 20$ for individuals with 1 to 6 years of education, and $13 / 14$ for illiterate individuals. Therefore, we also used a cutoff point of 22/23 (which we used as Method 2 in our study) for MoCA scores [24, 31, 37].

2.4. Water-Swallowing Test. The water-swallowing test (WST) is frequently used in clinical practice as a functional assessment to evaluate swallowing function [38]. Swallowing performance was assessed with the $30 \mathrm{~mL}$ water swallowing test which is cheap, easy to use, and with the highest reliability [39]. A total of $30 \mathrm{~mL}$ water was put on a plastic cup. The patient was ordered to drink the water "as quickly as comfortably possible" in an upright seated position. The time to drink and presence or absence of coughing were recorded. The results included the following five levels: level I (drink
TABLE 1: Demographics of the study participants.

\begin{tabular}{|c|c|c|c|c|}
\hline \multirow[b]{2}{*}{ Characteristics } & \multicolumn{2}{|c|}{ ALDH2 genotypes } & \multirow[b]{2}{*}{$\begin{array}{c}t / \text { chi } \\
\text { square }\end{array}$} & \multirow[b]{2}{*}{$\begin{array}{c}p \\
\text { value }\end{array}$} \\
\hline & $\begin{array}{c}* 1 / * 1 \\
(n=87)\end{array}$ & $\begin{array}{c}* 1 / * 2+* 2 / * 2 \\
(n=93)\end{array}$ & & \\
\hline Age (years) & $67.92 \pm 8.02$ & $69.88 \pm 9.53$ & 1.490 & 0.138 \\
\hline Males, $n(\%)$ & $38(43.7 \%)$ & $49(52.7 \%)$ & 8.536 & 0.003 \\
\hline Education & & & 7.013 & 0.071 \\
\hline$<6$ years & $18(20.7 \%)$ & $22(23.7 \%)$ & & \\
\hline $6-9$ years & $27(31.0 \%)$ & $19(20.4 \%)$ & & \\
\hline $9-12$ years & $21(24.1 \%)$ & $37(39.8 \%)$ & & \\
\hline$>12$ years & $21(24.1 \%)$ & $15(16.1 \%)$ & & \\
\hline Alcohol consumption & & & 12.464 & 0.006 \\
\hline Nondrinkers & $12.6 \%$ & $21.5 \%$ & & \\
\hline Light drinkers & $8.0 \%$ & $22.6 \%$ & & \\
\hline Moderate drinkers & $36.8 \%$ & $30.1 \%$ & & \\
\hline Excessive drinkers & $42.5 \%$ & $25.8 \%$ & & \\
\hline
\end{tabular}

Continuous variables are expressed as the mean \pm standard deviation when normally distributed, and categorical variables are expressed as percentages.

once, no coughing), level II (drinking more than two times of interruption, no coughing), level III (drinking once, with coughing), level IV (drinking more than two times of interruption, with coughing), and level V (coughing frequently and cannot drink the water successfully). After examination, swallowing ability was classified as normal (level I within $5 \mathrm{~s}$ ), possible abnormality (level I over $5 \mathrm{~s}$ or level II), and abnormality (levels III to V). Possible abnormality and abnormality are considered dysphagia [39].

2.5. Alcohol Consumption Measurements. Data regarding alcohol consumption were collected via a self-administered questionnaire [40]. The questionnaire included a range of drinking variables in the past 12 months before the stroke. The type of alcohol (liquor, beer, or wine), quantity of consumption, and frequency of consumption (never or occasionally, daily, weekly, or monthly) were all assessed. The average daily intake of absolute alcohol was estimated based on the quantity and frequency of consumption. The content of ethanol (pure alcohol) was assumed to be $15.1 \mathrm{~g}$ for a drink of liquor, $13.2 \mathrm{~g}$ for a can of beer, and $10.8 \mathrm{~g}$ for a standard glass of wine $[41,42]$. For each participant, total ethanol intake was converted to standard units per week ( 1 unit $=8 \mathrm{~g}$ ethanol). Each participant's drinking status was then classified as one of four distinct categories: nondrinker, light drinker, moderate drinker, or excessive drinker [41]. Nondrinkers consumed $<1$ unit of ethanol per week. Light drinkers consumed 1-10 units/week for men and 1-7 units/week for women. Moderate drinkers consumed 11-21 units/week for men and 8-14 units/week for women. Excessive drinkers consumed $>21$ units/week for men and >14 units/week for women.

2.6. 4-HNE Concentration Measurements. The plasma levels of 4-HNE were estimated using ELISA kits (Elabscience Biotechnology, Wuhan, China) according to the manufacturer's instructions [21]. 
2.7. ALDH2 Genotyping Measurements. Genomic DNA samples were obtained from blood samples using commercial DNA extraction kits (Tiangen Biotech, Beijing, China). The primer sequences were as follows: forward primer, $5^{\prime}$ -GTCAACTGCTATGATGTGTTTGG- $3^{\prime}$ and reverse primer, $5^{\prime}$-CCACCAGCAGACCCTCAAG- $3{ }^{\prime}$. The $50 \mu \mathrm{L}$ polymerase chain reaction (PCR) mixture consisted of $2 \mu \mathrm{L}$ DNA template, $2 \mu \mathrm{L}$ forward and $2 \mu \mathrm{L}$ reverse primers, 25 $\mu \mathrm{L}$ TaqMan Master Mix, and $19 \mu \mathrm{L}$ double-distilled $\mathrm{H}_{2} \mathrm{O}$. The PCR was conducted with predenaturation for $3 \mathrm{~min}$ at $94^{\circ} \mathrm{C}$, followed by 35 cycles of amplification $\left(94^{\circ} \mathrm{C}\right.$ for $45 \mathrm{~s}$, $53^{\circ} \mathrm{C}$ for $30 \mathrm{~s}$, and $72^{\circ} \mathrm{C}$ for $45 \mathrm{~s}$ ), and extension for $5 \mathrm{~min}$ at $72^{\circ} \mathrm{C}[20]$. The PCR products were purified using commercial kits (Axygen Biosciences, Corning, NY, USA) and sent to GenScript Corporation (Nanjing, China) for sequencing.

2.8. Statistical Analyses. The results of continuous variables are presented as the mean \pm standard deviation $(\mathrm{SD})$, whereas the results of categorical variables are expressed as numbers of patients and percentages. Two-tailed Student's $t$ -test or one-way ANOVA was performed for continuous variables, and the $\chi^{2}$ test was performed for categorical variables. Binary logistic regression analysis was performed to determine the associations of $A L D H 2$ polymorphisms and alcohol consumption with cognitive impairment and swallowing ability after ischemic stroke in a Han Chinese population by estimating the odds ratios (ORs) with $95 \%$ confidence intervals (CIs) [43]. We used two different cognitive impairment assessment methods (Method 1 and Method 2) to estimate the correlations between $A L D H 2$ polymorphism, alcohol consumption, and cognitive impairment. All missing values of predictors were imputed. Statistical analyses were conducted using SPSS version 24.0 software (IBM Corporation, Chicago, USA). All $p$ values of less than 0.05 were taken as statistically significant.

\section{Results}

3.1. Baseline Characteristics. Table 1 shows the demographic characteristics of all participants, grouped by ALDH2 polymorphism. We combined the heterozygotes $(\mathrm{ALDH} 2 * 1 / * 2)$ and mutant homozygotes (ALDH2 $* 2 / * 2$ ) into one category and compared them with the wild-type homozygotes $(\mathrm{ALDH} 2 * 1 / * 1)$ in our analyses. There were no significant differences between the two categories in age or education. However, the levels of alcohol consumption were significantly higher in the ALDH2 wild-type genotype group than in the mutant and heterozygous genotype group $(p=0.006)$. We further compared alcohol consumption between two ALDH2 genotypes by gender; the results showed that there was no statistical difference between genotype and alcohol consumption in males. However, the levels of alcohol consumption were significantly higher in the ALDH2 wild-type genotype group than in the mutant and heterozygous genotype group in females $(p=0.001$, Supplementary Table 1).

3.2. Clinical Characteristics. The clinical characteristics of participants according to ALDH2 polymorphism are shown
TABle 2: Baseline characteristics of ischemic stroke patients with different $A L D H 2$ genotypes.

\begin{tabular}{|c|c|c|c|c|}
\hline \multirow[b]{2}{*}{ Characteristics } & \multicolumn{2}{|c|}{ ALDH2 genotypes } & \multirow[b]{2}{*}{$t$} & \multirow[b]{2}{*}{$p$ value } \\
\hline & $* 1 / * 1(n=87)$ & $\begin{array}{c}* 1 / * 2+* 2 / * 2 \\
(n=93)\end{array}$ & & \\
\hline BMI $\left(\mathrm{kg} / \mathrm{m}^{2}\right)$ & $24.46 \pm 2.91$ & $25.22 \pm 2.67$ & 1.821 & 0.070 \\
\hline SBP (mmHg) & $137.25 \pm 20.10$ & $142.65 \pm 24.83$ & 1.595 & 0.113 \\
\hline DBP (mmHg) & $81.95 \pm 9.24$ & $82.09 \pm 8.84$ & 0.098 & 0.922 \\
\hline $\mathrm{FPG}(\mathrm{mmol} / \mathrm{L})$ & $7.31 \pm 1.91$ & $7.58 \pm 1.98$ & 0.911 & 0.363 \\
\hline TC (mmol/L) & $6.16 \pm 1.94$ & $5.62 \pm 1.40$ & 2.106 & 0.037 \\
\hline $\mathrm{TG}(\mathrm{mmol} / \mathrm{L})$ & $2.13 \pm 0.65$ & $1.50 \pm 0.65$ & 1.252 & 0.214 \\
\hline $\mathrm{HDL}-\mathrm{C}(\mathrm{mmol} / \mathrm{L})$ & $1.18 \pm 0.37$ & $1.15 \pm 0.32$ & 0.583 & 0.561 \\
\hline LDL-C (mmol/L) & $2.51 \pm 0.96$ & $2.54 \pm 0.75$ & 0.234 & 0.815 \\
\hline 4-HNE (ng/mL) & $12.18 \pm 1.94$ & $13.42 \pm 2.11$ & 4.096 & 0.001 \\
\hline
\end{tabular}

Continuous variables are expressed as the mean \pm standard deviation when normally distributed, and categorical variables are expressed as percentages. BMI: body mass index; SBP: systolic blood pressure; DBP: diastolic blood pressure; FPG: fasting plasma glucose; TC: total cholesterol; TG: triglycerides; HDL-C: high-density lipoprotein cholesterol; LDL-C: low-density lipoprotein cholesterol; 4-HNE: 4-hydroxy-trans-2-nonenal.

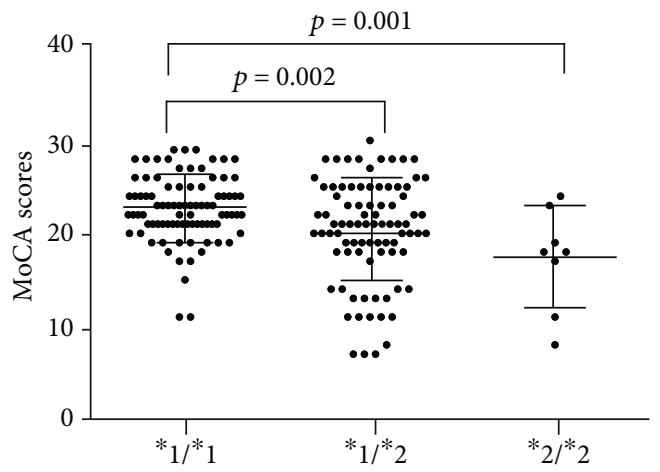

Figure 2: Comparison of Montreal Cognitive Assessment (MoCA) scores of ischemic stroke patients grouped by $A L D H 2$ genotype.

in Table 2. There were no significant differences between the two groups in BMI, systolic blood pressure (SBP), diastolic blood pressure (DBP), or FPG, TG, HDL-C, or LDL$\mathrm{C}$ levels. However, 4-HNE levels were higher in patients with mutant alleles $(13.42 \pm 2.11 \mathrm{ng} / \mathrm{L})$ than in patients with wildtype alleles $(12.18 \pm 1.94 \mathrm{ng} / \mathrm{L} ; p=0.001)$, whereas TC levels were lower in patients with mutant alleles $(5.62 \pm 1.40$ $\mathrm{mmol} / \mathrm{L})$ than in patients with wild-type alleles $(6.16 \pm 1.94$ $\mathrm{mmol} / \mathrm{L} ; p=0.037$ ).

3.3. Analysis of Cognitive Performance in Subjects with Different ALDH2 Genotypes. MoCA was used in ischemic stroke patients as a dependent variable to assess the extent of early vascular cognitive dysfunction. We compared the MoCA scores of three genotypes by analysis of variance $(F=8.643, p=0.0003)$. Figure 2 shows that the MoCA scores of the ALDH2 wild-type genotype group ( $n=87,22.64 \pm$ $3.55)$ were higher than those of the heterozygous group $(n=85,20.28 \pm 5.52 ; p=0.001)$. The MoCA scores of the 
TABLE 3: Comparison of alcohol consumption between the cognitive impairment and nonimpairment groups according to two different Montreal Cognitive Assessment (MoCA) cutoff scores.

\begin{tabular}{|c|c|c|c|c|}
\hline \multirow{2}{*}{ Alcohol consumption } & \multicolumn{2}{|c|}{ Method 1} & \multicolumn{2}{|c|}{ Method 2} \\
\hline & Impairment & Nonimpairment & Impairment & Nonimpairment \\
\hline Nondrinkers & $21(13.4 \%)$ & $10(43.5 \%)$ & $12(9.9 \%)$ & $19(32.2 \%)$ \\
\hline Light drinkers & $24(15.3 \%)$ & $4(17.4 \%)$ & $18(14.9 \%)$ & $10(16.9 \%)$ \\
\hline Moderate drinkers & $52(33.1 \%)$ & $8(34.8 \%)$ & $44(36.4 \%)$ & $16(27.1 \%)$ \\
\hline Excessive drinkers & $60(38.2 \%)$ & $1(4.3 \%)$ & $47(38.8 \%)$ & $14(23.7 \%)$ \\
\hline Chi square & 17.419 & & 15.238 & \\
\hline$p$ value & 0.001 & & 0.002 & \\
\hline
\end{tabular}

Method 1: MoCA cutoff score of 26. Method 2: MoCA cutoff score of 23.

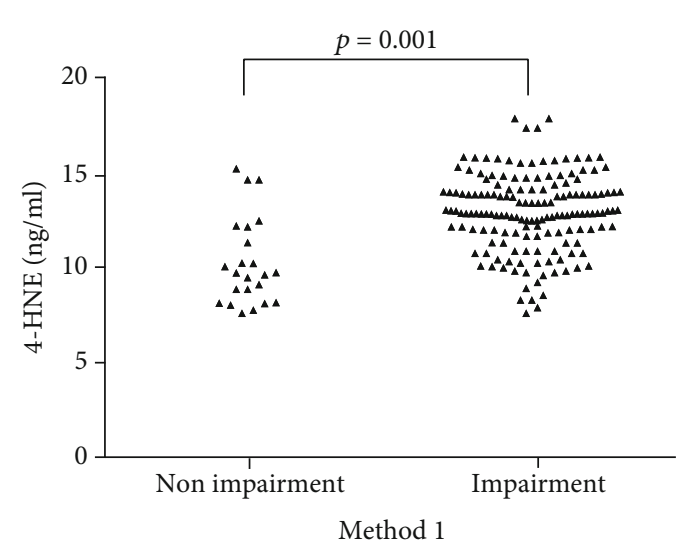

(a)

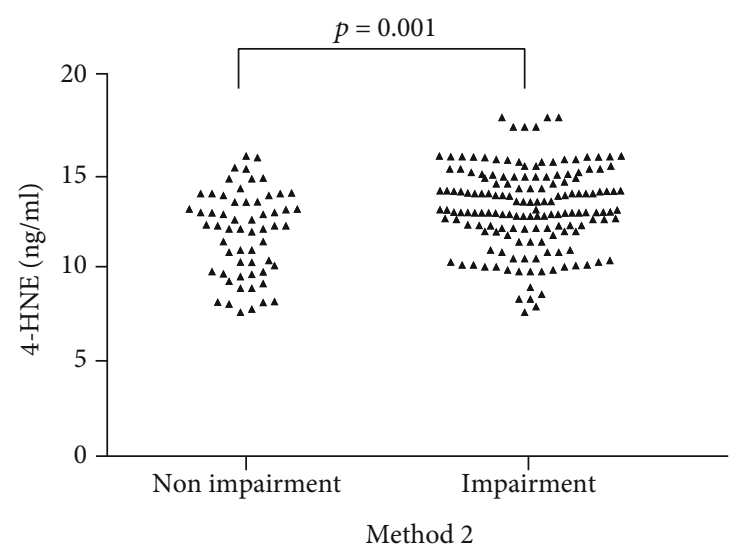

(b)

FIGURE 3: Comparison of 4-hydroxy-trans-2-nonenal (4-HNE) levels between the cognitive impairment and nonimpairment groups according to two different Montreal Cognitive Assessment (MoCA) cutoff scores: (a) score of 26; (b) score of 23.

ALDH2 wild-type genotype group (22.64 \pm 3.55$)$ were higher than that of the mutant genotype group $(n=8,17.25 \pm 5.45$; $p=0.002)$. And there was no difference between the heterozygous and mutant genotype groups $(p>0.05)$ (Figure 2$)$.

3.4. Association of Alcohol Consumption and 4-HNE Levels with Cognitive Impairment. We used two different cognitive impairment assessment methods to compare alcohol consumption between the cognitive impairment and nonimpairment groups (Table 3). For both of the MoCA cutoff scores, the percentage of moderate to excessive alcohol consumption was higher in the cognitive impairment group than in the nonimpairment group. According to the MoCA subscores of visuospatial/executive, naming, memory, attention, language, abstraction, delayed recall, and orientation, MoCA subscores were compared with alcohol consumption in two $A L D H 2$ genotypes. Intergroup differences were assessed using the single factor analysis of variance. Among the seven subscores, we found that two subscores (language and delayed recall) have a significant difference in alcohol consumption using univariate analysis (Supplementary Table 2).

We also used the two different MoCA cutoff scores to compare 4-HNE levels between the cognitive impairment and nonimpairment groups (Figure 3(a)). With a cutoff score of 26 , the levels of 4 -HNE were higher in the cognitive impairment group $(12.94 \pm 2.01, n=157)$ than in the nonim- pairment group $(10.29 \pm 2.29, n=23)$. Furthermore, when we considered MoCA scores and educational levels of the ischemic stroke patients, with a cutoff score of 23 (Figure 3(b)), the levels of 4-HNE were also higher in the cognitive impairment group $(12.98 \pm 2.14, n=121)$ than in the nonimpairment group $(11.81 \pm 2.22, n=59)$.

3.5. Association between Swallowing Function and Cognitive Impairment. We used two different cognitive impairment assessment methods, to compare swallowing levels between the cognitive impairment and nonimpairment groups (Table 4). For both of the MoCA cutoff scores, the level of swallowing function was higher in the cognitive impairment group than in the nonimpairment group.

3.6. Separate Effects of ALDH2 Polymorphisms and Alcohol Consumption on Cognitive Impairment. To test the possible association of $A L D H 2$ polymorphism and alcohol consumption with cognitive impairment, we assessed the separate effects of $A L D H 2$ polymorphism and alcohol consumption on cognitive impairment in ischemic stroke patients. The association between $A L D H 2$ polymorphisms and cognitive impairment risk in ischemic stroke patients is shown in Table 5. At a cutoff MoCA score of 26, participants carrying the mutant $A L D H 2$ allele had a higher risk of cognitive impairment $(\mathrm{OR}=3.29,95 \% \mathrm{CI}=1.03-10.50, p<0.05)$. 
TABLE 4: Comparison of swallowing function between the cognitive impairment and nonimpairment groups according to two different MoCA cutoff scores.

\begin{tabular}{|c|c|c|c|c|c|c|}
\hline \multirow{2}{*}{ Swallowing } & \multicolumn{2}{|c|}{ Method 1} & \multirow{2}{*}{$p$} & \multicolumn{2}{|c|}{ Method 2} & \multirow{2}{*}{$p$} \\
\hline & Impairment & Nonimpairment & & Impairment & Nonimpairment & \\
\hline Level I & $9(5.7 \%)$ & $23(100.0 \%)$ & 0.001 & $0(0.0 \%)$ & $32(54.2 \%)$ & 0.001 \\
\hline Level II & $44(28.0 \%)$ & $0(0.0 \%)$ & & $17(14.0 \%)$ & $27(45.8 \%)$ & \\
\hline Level III & $53(33.8 \%)$ & $0(0.0 \%)$ & & $53(43.8 \%)$ & $0(0.0 \%)$ & \\
\hline Level IV & $29(18.5 \%)$ & $0(0.0 \%)$ & & $29(24.0 \%)$ & $0(0.0 \%)$ & \\
\hline Level V & $22(14.0 \%)$ & $0(0.0 \%)$ & & $22(18.2 \%)$ & $0(0.0 \%)$ & \\
\hline
\end{tabular}

TABLE 5: Adjusted odds ratios (95\% confidence intervals) ${ }^{\text {a }}$ for the separate effects of ALDH2 polymorphism and alcohol consumption on cognitive impairment in ischemic stroke patients.

\begin{tabular}{lcc}
\hline & Method 1 & Method 2 \\
\hline $\begin{array}{l}\text { ALDH2 genotypes } \\
* 1 / * 1\end{array}$ & Ref & Ref \\
$* 1 / * 2+* 2 / * 2$ & $3.29(1.03-10.50)^{*}$ & $2.65(1.19-5.92)^{*}$ \\
Alcohol consumption & & \\
Nondrinkers & Ref & Ref \\
Light drinkers & $0.59(0.16-0.89)^{\#}$ & $0.68(0.33-0.89)^{\#}$ \\
Moderate drinkers & $0.77(0.21-1.06)$ & $1.08(0.83-2.19)$ \\
Excessive drinkers & $1.22(1.16-1.49)^{\#}$ & $1.78(1.03-2.15)^{\#}$ \\
\hline
\end{tabular}

${ }^{a}$ Models were adjusted for age, gender, and education. ${ }^{*} p<0.05$ vs. ALDH2 wild-type genotype group (ALDH2 $* 1 / * 1) .{ }^{*} p<0.05$ vs. nondrinkers.

Similarly, at a cutoff MoCA score of 23, participants carrying the mutant $A L D H 2$ allele also had a higher risk of cognitive impairment $(\mathrm{OR}=2.65,95 \% \mathrm{CI}=1.19-5.92, p<0.05)$.

Further analysis of these results revealed a clear association between alcohol consumption and cognitive impairment risk in ischemic stroke patients. At a MoCA cutoff score of 26 , there was an OR of $0.59(95 \% \mathrm{CI}=0.16-0.89)$ in light drinkers and an OR of $1.22(95 \% \mathrm{CI}=1.16-1.49)$ in excessive drinkers compared with nondrinkers. The OR values using a MoCA cutoff score of 23 were similar to the values using a cutoff score of 26 .

3.7. ORs of Alcohol Consumption on Cognitive Impairment, Stratified by ALDH2 Polymorphism. Table 6 shows the ORs of alcohol consumption on cognitive impairment, stratified by ALDH2 polymorphism, after taking into account other potential risk factors. At a MoCA cutoff score of 26, the multivariate OR of cognitive impairment risk was 7.75 (95\% CI: 1.03-113.78) for the $A L D H 2$ wild-type genotype in excessive drinkers compared with nondrinkers. In patients with the $A L D H 2$ heterozygous group, the multivariate OR (95\% CI) of cognitive impairment risk compared with nondrinkers was 10.95 (1.04-114.88) in moderate drinkers. The OR values had a similar trend using a cutoff MoCA score of 23. However, in patients with the $A L D H 2$ heterozygous group, the multivariate OR (95\% CI) of cognitive impairment risk compared with nondrinkers was 13.74 (1.96-96.51) in light drinkers, 22.36 (3.69-135.54) in moderate drinkers, and 20.93 (2.77-158.45) in excessive drinkers.

\section{Discussion}

Previous studies have revealed that $A L D H 2$ polymorphisms are closely related to the incidence of ischemic stroke. The current study was the first case-cohort study describing the effects of $A L D H 2$ polymorphisms and alcohol consumption on cognitive impairment after ischemic stroke. We demonstrated that $A L D H 2$ polymorphisms and alcohol consumption had a synergistic effect on cognitive impairment, even after taking other potential risk factors into account (age, gender, education, and subtype). Our results indicate that the association between alcohol consumption and cognitive impairment is stronger in the $A L D H 2$ heterozygous group than in the wild-type genotype group, as well as with swallowing ability.

Cognitive impairment after stroke can affect the quality of life and long-term prognosis (higher mortality and more disability) of stroke survivors [44]. Several studies have confirmed that the common functional single nucleotide polymorphism (SNP) in exon 12 of $A L D H 2$ is a risk indicator for ischemic stroke [21]. SNPs are the most abundant and stable genetic variations that exist in genomes $[45,46]$. In the present study, the $A L D H 2 * 2$ polymorphism was associated with cognitive impairment after ischemic stroke, although there was a lack of evidence of this in previous studies. Our data revealed that patients in the ALDH2 wild-type genotype group were significantly more likely to have higher MoCA scores than patients in the mutant and heterozygous genotype group, which suggests that the $A L D H 2 * 2$ polymorphism is associated with cognitive impairment after stroke.

The MoCA can be used as a dependent variable to assess the extent of early cognitive dysfunction [22]. In previous studies, cognitive impairment was defined by a MoCA cutoff score of $<26[23,32]$. However, some researchers have recommended that a cutoff score of $22 / 23$ points might be more suitable to detect cognitive impairment [24, 31, 37]. To test the possible association between $A L D H 2$ polymorphisms and cognitive impairment in patients with ischemic stroke, we therefore used two different MoCA cutoff scores to investigate interaction effects. We found that the $A L D H 2$ mutant allele carried a higher risk of cognitive impairment using both MoCA cutoff scores. Furthermore, 4-HNE levels were higher in the cognitive impairment group than in the nonimpairment group using both MoCA cutoff scores.

Several studies have demonstrated that 4-HNE is a potential substrate for ALDH2. The levels of 4-HNE are 
TABLE 6: Multivariate odds ratios (95\% confidence intervals) ${ }^{\mathrm{a}}$ for alcohol consumption on cognitive impairment in stroke patients, stratified by $A L D H 2$ polymorphism.

\begin{tabular}{lccc}
\hline ALDH2 genotypes & Alcohol consumption & Method 1 & Method 2 \\
\hline & Nondrinkers & Ref & $0.45(0.05-3.91)$ \\
$* 1 / * 1$ & Light drinkers & $1.75(0.20-15.19)$ & $2.43(0.54-11.02)$ \\
& Moderate drinkers & $2.97(0.60-14.68)$ & $3.93(0.84-18.44)$ \\
\hline & Excessive drinkers & $7.75(1.03-113.78)^{*}$ & Ref \\
$* 1 / * 2$ & Nondrinkers & Ref & $13.74(1.96-96.51)^{\#}$ \\
& Light drinkers & $15.99(0.96-266.02)$ & $22.36(3.69-135.54)^{\#}$ \\
& Moderate drinkers & $10.95(1.04-114.88)^{\#}$ & $20.93(2.77-158.45)^{\#}$ \\
\hline$* 2 / * 2$ & Excessive drinkers & - & - \\
& Nondrinkers & - & - \\
& Light drinkers & - & - \\
\hline
\end{tabular}

${ }^{\mathrm{a}}$ Models were adjusted for age, gender, education, and subtype. ${ }^{*} p<0.05$ vs. nondrinkers with ALDH2 wild-type genotype (ALDH2 $\left.* 1 / * 1\right) .{ }^{*} p<0.05$ vs. nondrinkers with ALDH2 mutant genotype (ALDH2 $* 1 / * 2$ ).

elevated following ischemic stroke injury. Guo et al. reported that 4-HNE plays an important role in the pathogenesis of neurological diseases and is a potential biomarker for ischemic stroke $[15,16]$. Our data revealed that 4-HNE levels were significantly lower in patients with the $A L D H 2$ wild-type genotype than in patients carrying mutant $A L D H 2$ alleles. Meanwhile, patients with the $A L D H 2$ wild-type genotype were significantly more likely to have a higher MoCA score compared with those carrying mutant alleles. This finding may explain why the $A L D H 2$ mutant allele carries a significantly higher risk of cognitive impairment.

Swallowing deficits are also commonly reported in patients with ischemic stroke [25]. Several studies revealed that cognitive dysfunction was associated with dysphagia $[25,26]$. Therefore, we investigated the association between swallowing function and cognitive impairment using two MoCA cutoff scores. In this study, the severity of dysphagia might contribute to cognitive impairment for both MoCA cutoff scores. In addition, considering that the lesion site may affect the swallowing function, we further adopted subtypes of ischemic stroke to investigate the interaction of ALDH2 and alcohol consumption on swallowing. Our data described that both $A L D H 2$ genotypes showed a higher risk of dysphagia in excessive drinkers compared to nondrinkers, but the risk of dysphagia was higher in carriers of the mutant $A L D H 2$ allele than in noncarriers.

As an important determinant of drinking behavior, ALDH2 has a well-known role in ethanol metabolism [47]. Various longitudinal studies have reported a link between moderate alcohol consumption and improved cognitive performance $[48,49]$. In our study, we also explored the association between alcohol consumption and cognitive function in patients with ischemic stroke. Considerable evidence has emerged suggesting that alcohol consumption behaviors are related to the $A L D H 2 * 2$ polymorphism in Asian populations $[50,51]$. We divided the ischemic stroke patients in our study into four subgroups based on their history of alco- hol consumption. Alcohol consumption in the $A L D H 2$ wildtype genotype group was significantly higher than that in the mutant and heterozygous genotype group. This may be because carriers of $A L D H 2$ mutant alleles are more sensitive to alcohol, which reportedly makes them less inclined to engage in excessive drinking [52]. We used two different cognitive impairment cutoff points to analyze the association between alcohol consumption and cognitive impairment. The univariate analysis revealed a higher percentage of moderate to excessive alcohol consumption in the cognitive impairment group than in the nonimpairment group, which suggests that alcohol consumption may have an effect on cognitive impairment after ischemic stroke. We also applied a binary logistic regression model to evaluate the synergistic effects of $A L D H 2$ polymorphisms and alcohol consumption on cognitive impairment. Adjusted for age, gender, education, and subtype, we demonstrated that the multivariate risk of cognitive impairment was higher in excessive drinkers than in nondrinkers with the $A L D H 2$ wild-type genotype, while the risk of cognitive impairment was higher in light to excessive drinkers than nondrinkers with the $A L D H 2$ mutant or heterozygous genotype. These findings further suggest that ALDH2 might be involved in the pathogenesis and progression of cognitive impairment after ischemic stroke, as well as having a role in alcohol metabolism.

There were several limitations in the present study. First, some participants refused to participate, while blood samples were unable to be obtained from some patients; this may have caused sampling bias. Second, the MoCA scale is commonly used as a screening scale for cognitive function but fails to assess global disability after ischemic stroke. So, in this study, we also evaluated the swallowing function. The current findings are therefore considered preliminary and require validation. Third, the plasma 4-HNE levels were detected only once, at baseline, and potential fluctuations in plasma 4HNE levels were not evaluated; we were therefore unable to adjust for this effect. Finally, although the ALHD2 $* 2$ allele 
is an important risk factor for ischemic stroke, results have been inconsistent over different ethnic groups, different countries, and different genders. Because the sample size was relatively small, we did not perform a stratified analysis by lesion location. We adopted subtypes of ischemic stroke to replace lesion location, and this might have affected our study results (Supplementary Table 3). To better understand the relationship between ischemic stroke impairment and $A L D H 2$ genotypes, future studies need to enroll larger sample sizes across multiple communities. To this end, the current study represents an ongoing effort, and we will continue to regularly update the analysis, with the aim of providing a comprehensive and easily accessible review, as well as facilitating a best-practice approach to ischemic stroke rehabilitation.

\section{Conclusions}

The present study demonstrated that ALDH2 polymorphisms and alcohol consumption were associated with cognitive impairment and dysphagia in patients after ischemic stroke, mainly in patients with the mutant allele. ALDH2 may be involved in the pathogenesis and progression of ischemic stroke in the Han Chinese population. ALDH2 might therefore be a useful biomarker to target for cognitive rehabilitation following ischemic stroke. However, the underlying mechanisms need to be further explored.

\section{Data Availability}

The datasets used and/or analyzed during the current study are available from the corresponding author on reasonable request.

\section{Ethical Approval}

This study was conducted with approval from the Ethics Committee of Bengbu Medical College.

\section{Disclosure}

The authors are accountable for all aspects of the work in ensuring that questions related to the accuracy or integrity of any part of the work are appropriately investigated and resolved.

\section{Conflicts of Interest}

The authors declare that there is no conflict of interest regarding the publication of this paper.

\section{Acknowledgments}

This work was supported by the Centers for Disease Control and Prevention of Bengbu, China. The work was also supported by the Natural Science Foundation of the Education Department of Anhui Province (KJ2019A0306), the Transformation of Medicine Research Projects of Bengbu Medical College (BYTM2019004), the 512 Talent Cultivation Plan of Bengbu Medical College (by51201202 and by51201307), and the Chronic Noncommunicable Disease Prevention and Control Program of Bengbu Medical College (BYKC201901), China. We are grateful to all of the professionals and participants involved in the study. We also thank Bronwen Gardner, PhD, from Liwen Bianji, Edanz Editing China (http:// www.liwenbianji.cn/ac), for editing the English text of a draft of this manuscript.

\section{Supplementary Materials}

Supplementary Table 1: comparison of MoCA subscores based on ALDH2 polymorphisms and alcohol consumption. Supplementary Table 2: comparison of MoCA subscores based on ALDH2 polymorphisms and alcohol consumption. Supplementary Table 3: multivariate odds ratios (95\% confidence intervals) for alcohol consumption on swallowing ability in stroke patients, stratified by ALDH2 polymorphism. (Supplementary Materials)

\section{References}

[1] W. J. Tu, H. C. Qiu, Y. Zhang et al., "Lower serum retinoic acid level for prediction of higher risk of mortality in ischemic stroke," Neurology, vol. 92, no. 15, pp. e1678-e1687, 2019.

[2] W. J. Tu, X. Dong, S. J. Zhao, D.-G. Yang, and H. Chen, "Prognostic value of plasma neuroendocrine biomarkers in patients with acute ischaemic stroke," Journal of Neuroendocrinology, vol. 25, no. 9, pp. 771-778, 2013.

[3] W. Johnson, O. Onuma, M. Owolabi, and S. Sachdev, "Stroke: a global response is needed," Bulletin of the World Health Organization, vol. 94, no. 9, pp. 634-634A, 2016.

[4] Y. Qu, L. Zhuo, N. Li et al., "Prevalence of post-stroke cognitive impairment in china: a community-based, crosssectional study," PLoS One, vol. 10, no. 4, p. e0122864, 2015.

[5] K. Tokairin, T. Osanai, T. Abumiya, K. Kazumata, K. Ono, and K. Houkin, "Regional transarterial hypothermic infusion in combination with endovascular thrombectomy in acute ischaemic stroke with cerebral main arterial occlusion: protocol to investigate safety of the clinical trial," BMJ Open, vol. 7, no. 8, p. e016502, 2017.

[6] C.-T. Yao, C.-A. Cheng, H.-K. Wang et al., "The role of $\mathrm{ALDH} 2$ and ADH1B polymorphism in alcohol consumption and stroke in Han Chinese," Hum Genomics, vol. 5, no. 6, pp. 569-576, 2011.

[7] Y. L. Zheng, F. Lian, Q. Shi et al., "Alcohol intake and associated risk of major cardiovascular outcomes in women compared with men: a systematic review and meta-analysis of prospective observational studies," BMC Public Health, vol. 15, no. 1, p. 773, 2015.

[8] J. Patra, B. Taylor, H. Irving et al., "Alcohol consumption and the risk of morbidity and mortality for different stroke types-a systematic review and meta-analysis," BMC Public Health, vol. 10, no. 1, p. 258, 2010.

[9] C. Zhang, Y. Y. Qin, Q. Chen et al., "Alcohol intake and risk of stroke: A dose-response meta-analysis of prospective studies," International Journal of Cardiology, vol. 174, no. 3, pp. 669677, 2014.

[10] M. V. Holmes, C. E. Dale, L. Zuccolo et al., "Association between $\mathrm{C}$ reactive protein and coronary heart disease: mendelian randomisation analysis based on individual participant data," BMJ, vol. 349, p. g4164, 2014. 
[11] I. Y. Millwood, R. G. Walters, X. W. Mei et al., "Conventional and genetic evidence on alcohol and vascular disease aetiology: a prospective study of 500000 men and women in China," The Lancet, vol. 393, no. 10183, pp. 1831-1842, 2019.

[12] A. M. Wood, S. Kaptoge, A. S. Butterworth et al., "Risk thresholds for alcohol consumption: combined analysis of individual-participant data for 599912 current drinkers in 83 prospective studies," The Lancet, vol. 391, no. 10129, pp. 1513-1523, 2018.

[13] I. Lang, R. B. Wallace, F. A. Huppert, and D. Melzer, "Moderate alcohol consumption in older adults is associated with better cognition and well-being than abstinence," Age Ageing, vol. 36, no. 3, pp. 256-261, 2007.

[14] P. J. Brooks, M.-A. Enoch, D. Goldman, T.-K. Li, and A. Yokoyama, "The alcohol flushing response: an unrecognized risk dactor for esophageal cancer from alcohol consumption," PLoS Medicine, vol. 6, no. 3, pp. 258-263, 2009.

[15] J. M. Guo, A. J. Liu, P. Zang et al., "ALDH2 protects against stroke by clearing 4-HNE," Cell Research, vol. 23, no. 7, pp. 915-930, 2013.

[16] W. C. Lee, H. Y. Wong, Y. Y. Chai et al., "Lipid peroxidation dysregulation in ischemic stroke: plasma 4-HNE as a potential biomarker?," Biochemical and Biophysical Research Communications, vol. 425, no. 4, pp. 842-847, 2012.

[17] C. H. Chen, G. R. Budas, E. N. Churchill, M.-H. Disatnik, T. D. Hurley, and D. Mochly-Rosen, "Activation of aldehyde dehydrogenase-2 reduces ischemic damage to the heart," Science, vol. 321, no. 5895, pp. 1493-1495, 2008.

[18] M. J. Stewart, K. Malek, and D. W. Crabb, "Distribution of messenger RNAs for aldehyde dehydrogenase 1, aldehyde dehydrogenase 2, and aldehyde dehydrogenase 5 in human tissues," Journal of investigative medicine: the official publication of the American Federation for Clinical Research, vol. 44, no. 2, pp. 42-46, 1996.

[19] Y. Qu, H. L. Zhang, L. M. Yu, Y. Sun, H.-l. Wu, and Y.-g. Chen, "Aldehyde dehydrogenase 2 polymorphism as a protective factor for intracranial vascular stenosis in ischemic stroke in Han Chinese," International Journal of Neuroscience, vol. 126, no. 4, pp. 342-347, 2016.

[20] F. Xu, Y. Chen, R. Lv et al., "ALDH2 genetic polymorphism and the risk of type II diabetes mellitus in CAD patients," Hypertens Res, vol. 33, no. 1, pp. 49-55, 2010.

[21] H. Yang, Z. Song, G. P. Yang et al., "The ALDH2 rs671 polymorphism affects post-stroke epilepsy susceptibility and plasma 4-HNE levels," PLoS One, vol. 9, no. 10, p. e109634, 2014.

[22] L. Zuo, Y. Dong, R. Zhu et al., "Screening for cognitive impairment with the Montreal Cognitive Assessment in Chinese patients with acute mild stroke and transient ischaemic attack: a validation study," BMJ Open, vol. 6, no. 7, p. e011310, 2016.

[23] L. Chen, C. Yu, N. Zhang, J. Liu, and W. Liu, "Cognitive impairment in patients with Parkinson's disease: a 30-month follow-up study," Clinical neurology and neurosurgery, vol. 151, pp. 65-69, 2016.

[24] N. Carson, L. Leach, and K. J. Murphy, "A re-examination of Montreal Cognitive Assessment (MoCA) cutoff scores," International journal of geriatric psychiatry, vol. 33, no. 2, pp. 379388, 2018.

[25] M. A. Crary, G. D. Carnaby, I. Sia, A. Khanna, and M. F. Waters, "Spontaneous swallowing frequency has potential to identify dysphagia in acute stroke," Stroke, vol. 44, no. 12, pp. 3452-3457, 2013.
[26] S. Y. Jo, J.-W. Hwang, and S.-B. Pyun, "Relationship between cognitive function and dysphagia after stroke," Annals of rehabilitation medicine, vol. 41, no. 4, p. 564, 2017.

[27] G. Wang, X. Cheng, and X. Zhang, "Use of various CT imaging methods for diagnosis of acute ischemic cerebrovascular disease," Neural regeneration research, vol. 8, no. 7, p. 655, 2013.

[28] Y. Yamada, J. Sakuma, I. Takeuchi et al., "Identification of six polymorphisms as novel susceptibility loci for ischemic or hemorrhagic stroke by exome-wide association studies," International Journal of Molecular Medicine, vol. 39, no. 6, pp. 1477-1491, 2017.

[29] W.-D. Tao, M. Liu, M. Fisher et al., "Posterior versus anterior circulation infarction: how different are the neurological deficits?," Stroke, vol. 43, no. 8, pp. 2060-2065, 2012.

[30] Q. Zeng, W. Tao, C. Lei, W. Dong, and M. Liu, "Etiology and risk factors of posterior circulation infarction compared with anterior circulation infarction," Journal of Stroke and Cerebrovascular Diseases, vol. 24, no. 7, pp. 1614-1620, 2015.

[31] K. L. Chen, Y. Xu, A. Q. Chu et al., "Validation of the Chinese version of Montreal Cognitive Assessment basic for screening mild cognitive impairment," Journal of the American Geriatrics Society, vol. 64, no. 12, pp. e285-e290, 2016.

[32] Z. S. Nasreddine, N. A. Phillips, V. Bédirian et al., "The Montreal Cognitive Assessment, MoCA: a brief screening tool for mild cognitive impairment," Journal of the American Geriatrics Society, vol. 53, no. 4, pp. 695-699, 2005.

[33] M. Ihara, Y. Okamoto, and R. Takahashi, "Suitability of the Montreal cognitive assessment versus the mini-mental state examination in detecting vascular cognitive impairment," Journal of Stroke and Cerebrovascular Diseases, vol. 22, no. 6, pp. 737-741, 2013.

[34] J. C. Davis, S. Bryan, L. C. Li et al., "Mobility and cognition are associated with wellbeing and health related quality of life among older adults: a cross-sectional analysis of the Vancouver Falls Prevention Cohort.," BMC Geriatrics, vol. 15, no. 1, p. $75,2015$.

[35] S. Freitas, M. R. Simoes, L. Alves, and I. Santana, "Montreal Cognitive Assessment (MoCA): normative study for the Portuguese population," Journal of Clinical and Experimental Neuropsychology, vol. 33, no. 9, pp. 989-996, 2011.

[36] L. Zhang, "Determination of the cut-off point of the Chinese version of the Montreal Cognitive Assessment among Chinese elderly in Guangzhou," Chinese Mental Health Journal, vol. 22, no. 2, p. 123, 2008.

[37] M. Nara, M. Sugie, T. Takahashi et al., "Japanese version of the Montreal Cognitive Assessment cut-off score to clarify improvement of mild cognitive impairment after exercise training in community-dwelling older adults," Geriatrics \& gerontology international, vol. 18, no. 6, pp. 833-838, 2018.

[38] A. Osawa and S. Maeshima, "Swallowing Disorders in Patients with Stroke," in Voice and Swallowing Disorders, IntechOpen., 2019.

[39] T. Wang, Y. Zhao, and A. Guo, "Association of swallowing problems with frailty in Chinese hospitalized older patients," International journal of nursing sciences, vol. 7, no. 4, pp. 408-412, 2020.

[40] I. Y. Millwood, L. Li, M. Smith et al., "Alcohol consumption in 0.5 million people from 10 diverse regions of China: prevalence, patterns and socio-demographic and health-related correlates," International journal of epidemiology, vol. 42, no. 3, pp. 816-827, 2013. 
[41] W. Huang, C. Qiu, B. Winblad, and L. Fratiglioni, "Alcohol consumption and incidence of dementia in a community sample aged 75 years and older," Journal of Clinical Epidemiology, vol. 55, no. 10, pp. 959-964, 2002.

[42] E. Glovannucci, G. Colditz, M. J. Stampfer et al., "The assessment of alcohol consumption by a simple self-administered questionnaire," American journal of epidemiology, vol. 133, no. 8, pp. 810-817, 1991.

[43] A. Jaywant, J. Toglia, F. M. Gunning, and M. W. O’Dell, “The diagnostic accuracy of the Montreal Cognitive Assessment in inpatient stroke rehabilitation," Neuropsychological Rehabilitation, vol. 29, no. 8, pp. 1163-1176, 2019.

[44] E. Douven, S. H. Schievink, F. R. Verhey et al., "The Cognition and Affect after Stroke - a Prospective Evaluation of Risks (CASPER) study: rationale and design," BMC Neurology, vol. 16, no. 1, p. 65, 2016.

[45] J. Zhang, J. Yang, L. Zhang et al., "A new SNP genotyping technology target SNP-seq and its application in genetic analysis of cucumber varieties," Scientific Reports, vol. 10, no. 1, p. 5623, 2020.

[46] B. Lehne, C. M. Lewis, and T. Schlitt, "From SNPs to genes: disease association at the gene level," PLoS One, vol. 6, no. 6, p. e20133, 2011.

[47] S. E. Luczak, T. Liang, and T. L. Wall, "Age of drinking initiation as a risk factor for alcohol use disorder symptoms is moderated by ALDH22 and ethnicity," Alcoholism: Clinical and Experimental Research, vol. 41, no. 10, pp. 1738-1744, 2017.

[48] G. E. Bond, R. L. Burr, S. M. McCurry, M. M. Rice, A. R. Borenstein, and E. B. Larson, "Alcohol and cognitive performance: a longitudinal study of older Japanese Americans. The Kame Project," International Psychogeriatrics, vol. 17, no. 4, pp. 653-668, 2005.

[49] K. J. Mukamal, L. H. Kuller, and A. L. Fitzpatrick, "Prospective study of alcohol consumption and risk of dementia in older adults," JAMA, vol. 289, no. 11, pp. 1405-1413, 2003.

[50] L. Wu, Y. He, B. Jiang et al., "The association between the prevalence, treatment and control of hypertension and the risk of mild cognitive impairment in an elderly urban population in China," Hypertension Research, vol. 39, no. 5, pp. 367-375, 2016.

[51] C. Shin, K. Kwack, N. H. Cho, S. H. Kim, and I. Baik, "Sex-specific differences in the association of a common aldehyde dehydrogenase 2 gene polymorphism and alcohol consumption with stroke risk in a Korean population: a prospective cohort study," Nutrition Research and Practice, vol. 9, no. 1, pp. 79-86, 2015.

[52] T. Takeshita and K. Morimoto, "Self-reported alcoholassociated symptoms and drinking behavior in three LDH2 genotypes among Japanese university students," Alcoholism: Clinical and Experimental Research, vol. 23, no. 6, pp. 10651069, 1999. 